\title{
Research Article \\ On the Local Discontinuous Galerkin Method for Linear Elasticity
}

\author{
Yuncheng Chen, ${ }^{1}$ Jianguo Huang, ${ }^{1,2}$ Xuehai Huang, ${ }^{1}$ \\ and Yifeng $X \mathbf{u}^{1}$ \\ ${ }^{1}$ Department of Mathematics, Shanghai Jiao Tong University, Shanghai 200240, China \\ ${ }^{2}$ Division of Computational Science, E-Institute of Shanghai Universities and Scientific Computing Key \\ Laboratory of Shanghai Universities, Shanghai Normal University, China
}

Correspondence should be addressed to Jianguo Huang, jghuang@sjtu.edu.cn

Received 25 February 2010; Accepted 22 May 2010

Academic Editor: Angelo Luongo

Copyright (C) 2010 Yuncheng Chen et al. This is an open access article distributed under the Creative Commons Attribution License, which permits unrestricted use, distribution, and reproduction in any medium, provided the original work is properly cited.

Following Castillo et al. (2000) and Cockburn (2003), a general framework of constructing discontinuous Galerkin (DG) methods is developed for solving the linear elasticity problem. The numerical traces are determined in view of a discrete stability identity, leading to a class of stable DG methods. A particular method, called the LDG method for linear elasticity, is studied in depth, which can be viewed as an extension of the LDG method discussed by Castillo et al. (2000) and Cockburn (2003). The error bounds in $L^{2}$-norm, $H^{1}$-norm, and a certain broken energy norm are obtained. Some numerical results are provided to confirm the convergence theory established.

\section{Introduction}

This paper is focused on systematically studying discontinuous Galerkin (DG) methods for the linear elasticity problem. Since the DG method was first introduced in 1970s, these methods have been applied for solving a variety of mathematical-physical problems including linear and nonlinear hyperbolic problems, Navier-Stokes equations, convection-dominated diffusion problems, and so on. The DG method may be viewed as high-order extensions of the classical finite volume method. Since no inter-element continuity is imposed, such methods can be defined on very general meshes including nonconforming meshes. Moreover, polynomials of arbitrary degree can be used on each element, making these methods suitable for $h p$-adaptivity. We refer to [1] for an excellent historical survey. As a generalization of the DG method proposed in [2] for the solution of the compressible Navier-Stokes equations, some local discontinuous Galerkin 
(LDG) methods were introduced and analyzed in [3,4] for time-dependent convectiondiffusion systems and second-order elliptic problems, respectively. After that, a unified theory was developed for conducting error analysis for DG methods of elliptic problems (cf. [5]). And a new framework was proposed in [6] for designing and analyzing DG methods, where stabilization mechanism frequently used in DG methods was also investigated. In [7], an important framework was proposed for constructing stable DG methods.

On the other hand, linear elasticity discusses how solid objects deform and become internally stressed on prescribed loading conditions, and is encountered extensively in structural analysis and engineering design. By use of usual displacement-based finiteelement methods for the elasticity equation, we are able to numerically determine the displacement field directly. However, in many engineering applications, the stress field is a quantity of more interest. For this, we may apply mixed finite-element methods to solve the linear elasticity system, from which the aforementioned two physical quantities can be obtained simultaneously. Over the past four decades, there have been many efforts along this line. But due to the symmetry constraint on the stress tensor, it is extremely difficult to construct stable stress-displacement finite elements. In two spatial dimensions, the first stable finite element with polynomial shape functions is presented in [8]. For the lowest-order element, the discrete stress space is composed of certain piecewise cubic polynomials, while the discrete displacement space consists of piecewise linear polynomials. In three dimensions, a piecewise quadratic stress space is constructed with 162 degrees of freedom on each tetrahedron (cf. [9]). Another approach in this direction is to use composite elements (macroelements), in which the discrete displacement space consists of piecewise polynomials with respect to one triangulation of the domain, while the discrete stress space consists of piecewise polynomials with respect to a more refined triangulation (cf. [10-13]). It is mentioned that for solving the previous problem, several mixed elements with weakly imposed symmetry have also been developed (cf. [1416]).

Regarding the complexity of mixed elements given above, the discontinuous Galerkin method is naturally a suitable alternative for numerically solving linear elasticity problems. To the best of our knowledge, a local DG method and an interior penalty DG method for linear elasticity are presented in [17] and [18, 19], respectively. A mixed DG method is given in [20], which one may find is covered by our general formulation below. Moreover, a mixed formulation is also extended to the case of nonsymmetric stress tensors (cf. [21]).

In this paper, we are going to look for new DG methods for the linear elasticity problem. Following [4, 7], we build up a framework to construct our DG methods. Then a discrete stability identity is established, from which we derive feasible choices of numerical traces and get a class of stable DG methods for linear elasticity. With a parameter taken to be zero, namely, $C_{22}=0$, we get an LDG method. Following [5] and using some technical arguments, we get optimal-order error estimates for the LDG method proposed in a certain broken energy norm, $H^{1}$-norm, and $L^{2}$-norm, respectively. It should be emphasized that in our formulation the symmetry of the stress tensor is preserved automatically.

The rest of this paper is organized as follows. The basic framework of DG methods and the determination of numerical traces are presented in Section 2. An error analysis for the LDG method is given in Section 3. And in Section 4, some numerical results are included to confirm our theoretical convergence orders. 


\section{The DG Method for Linear Elasticity}

\subsection{Basic Framework for the DG Method}

Assume that $\Omega \subset \mathbb{R}^{d}(d=2,3)$ is a bounded polygon or polyhedron. Let $\sigma=\left(\sigma_{i j}\right)_{d \times d}$ be the stress, let $\mathbf{u}=\left(u_{1}, \ldots, u_{d}\right)^{t}$ be the displacement, and let $\mathbf{f}=\left(f_{1}, \ldots, f_{d}\right)^{t}$ be the body force. Denote by $\varepsilon(\mathbf{u}):=\left(\varepsilon_{i j}(\mathbf{u})\right)_{d \times d}$ the linearized strain tensor with $\varepsilon_{i j}(\mathbf{u}):=\left(\partial u_{i} / \partial x_{j}+\partial u_{j} / \partial x_{i}\right) / 2$, tr the trace operator, and $\nabla \cdot$ the divergence operator. Then, the mathematical model of linear elasticity reads (cf. [22])

$$
\begin{gathered}
\mathcal{A} \boldsymbol{\sigma}-\boldsymbol{\varepsilon}(\mathbf{u})=\mathbf{0} \quad \text { in } \Omega, \\
-\nabla \cdot \boldsymbol{\sigma}=\mathbf{f} \quad \text { in } \Omega, \\
\mathbf{u}=\mathbf{0} \quad \text { on } \partial \Omega,
\end{gathered}
$$

where $A$ is the fourth-order compliance tensor defined by

$$
\mathcal{A} \sigma=\frac{1}{2 \mu}\left(\sigma-\frac{\lambda}{d \lambda+2 \mu}(\operatorname{tr} \sigma) \delta\right)
$$

where $\delta:=\left(\delta_{i j}\right)_{d \times d}$ is the Kronecker tensor, and the positive constants $\lambda$ and $\mu$ are the Lamé coefficients of the elastic material under consideration.

For defining our DG methods for problem (2.1), we introduce some notations first of all. For any Banach space $B$, the subspace of symmetric matrix-valued function is denoted by $(B)_{d \times d}^{s}$. Given a bounded domain $G \subset \mathbb{R}^{d}$ and a nonnegative integer $m$, let $H^{m}(G)$ be the usual Sobolev space consisting of all functions in $L^{2}(G)$ whose weak derivatives up to degree $m$ are also $L^{2}(G)$-integrable (cf. [23]). The corresponding norm is denoted by $\|\cdot\|_{m, G}$. Let $H_{0}^{m}(G)$ be the closure of $C_{0}^{\infty}(G)$ with respect to the norm $\|\cdot\|_{m, G}$. Moreover, we simply write $\|\cdot\|_{m}$ for $\|\cdot\|_{m, \Omega}$.

Let $\left\{\tau_{h}\right\}_{h>0}$ be a regular family of triangulations of $\Omega$ (cf. [23, 24]); $h:=\max _{K \in \tau_{h}} h_{K}$ and $h_{K}:=\operatorname{diam}(K)$. Let $\varepsilon_{h}$ be the union of all faces (edges) of the triangulation $\tau_{h}$ and $\varepsilon_{h}^{i}$ the union of all interior faces (edges) of the triangulation $\tau_{h}$. For any $e \in \varepsilon_{h}, h_{e}$ is set to be the diameter of $e$. Based on the triangulation $\tau_{h}$, let

$$
\begin{gathered}
\Sigma:=\left\{\left.\boldsymbol{\tau} \in\left(L^{2}(\Omega)\right)_{d \times d^{\prime}}^{s} \tau_{i j}\right|_{K} \in H^{1}(K), \forall K \in \tau_{h}, i, j=1, \ldots, d\right\}, \\
\mathbf{V}:=\left\{\mathbf{v} \in\left(L^{2}(\Omega)\right)^{d} ;\left.v_{i}\right|_{K} \in H^{1}(K), \forall K \in \tau_{h}, i=1, \ldots, d\right\} .
\end{gathered}
$$

The corresponding finite element spaces are given by

$$
\begin{gathered}
\boldsymbol{\Sigma}_{h}:=\left\{\boldsymbol{\tau} \in\left(L^{2}(\Omega)\right)_{d \times d^{\prime}}^{s} ;\left.\tau_{i j}\right|_{K} \in \mathcal{S}_{1}(K), \forall K \in \tau_{h}, i, j=1, \ldots, d\right\}, \\
\mathbf{V}_{h}:=\left\{\mathbf{v} \in\left(L^{2}(\Omega)\right)^{d} ;\left.v_{i}\right|_{K} \in \mathcal{S}_{2}(K), \forall K \in \tau_{h}, i=1, \ldots, d\right\},
\end{gathered}
$$


where, for each $K \in \tau_{h}, \mathcal{S}_{1}(K)$ and $\mathcal{S}_{2}(K)$ are two finite-dimensional spaces of polynomials in $K$ containing $P_{l}(K)$ and $P_{k}(K)$, respectively, with $k, l \geq 0$. Here, for a nonnegative integer $m, P_{m}(K)$ stands for the set of all polynomials in $K$ with the total degree no more than $m$.

assume that

To guarantee uniqueness of the solution to the LDG method to be proposed, we always

$$
\varepsilon\left(\mathbf{V}_{h}\right) \subset \Sigma_{h}, \quad \mathscr{A} \Sigma_{h} \subset \Sigma_{h}
$$

For a function $v \in L^{2}(\Omega)$ with $\left.v\right|_{K} \in H^{m}(K)$ for all $K \in \tau_{h}$, let $\|v\|_{m, h}$ be the usual broken $H^{m}$-type norm of $v$ defined by

$$
\|v\|_{m, h}=\left(\sum_{K \in \tau_{h}}\|v\|_{m, K}^{2}\right)^{1 / 2}
$$

If $\mathbf{v}$ is a vector-valued or matrix-valued function, the corresponding term $\|\mathbf{v}\|_{m, h}$ is defined in a similar manner. For a vector or a matrix $\mathbf{v}$, denote by $|\mathbf{v}|$ the quantity $(\mathbf{v} \cdot \mathbf{v})^{1 / 2}$ or $(\mathbf{v}$ : $\mathbf{v})^{1 / 2}$. Here the symbol : stands for the double dot product operation of matrices. Throughout this paper, we use the notation " $\lesssim \ldots$ " to indicate " $\leq C \cdots$ ", where $C$ is a generic positive constant independent of $h$ and other parameters, which may take different values at different appearances.

Let $K^{+}$and $K^{-}$be two adjacent elements of $\tau_{h}$. Let $\mathbf{x}$ be an arbitrary point of the set $e^{\prime}=\partial K^{+} \cap \partial K^{-}$, and let $\mathbf{n}^{+}$and $\mathbf{n}^{-}$be the corresponding outward unit normals at that point. For a vector-valued function $\mathbf{v}$ smooth inside each element $K^{ \pm}$, let us denote by $\mathbf{v}^{ \pm}$ the trace of $\mathbf{v}$ on $e^{\prime}$ from the interior of $K^{ \pm}$. Then we define averages and jumps at $\mathbf{x} \in e^{\prime}$ as follows:

$$
\begin{gathered}
\{p\}=\frac{1}{2}\left(p^{+}+p^{-}\right), \quad[p]=p^{+} \mathbf{n}^{+}+p^{-} \mathbf{n}^{-} \\
\{\mathbf{v}\}=\frac{1}{2}\left(\mathbf{v}^{+}+\mathbf{v}^{-}\right), \quad[\mathbf{v}]=\mathbf{v}^{+} \cdot \mathbf{n}^{+}+\mathbf{v}^{-} \cdot \mathbf{n}^{-}, \\
\{\boldsymbol{\tau}\}=\frac{1}{2}\left(\boldsymbol{\tau}^{+}+\boldsymbol{\tau}^{-}\right), \quad[\boldsymbol{\tau}]=\boldsymbol{\tau}^{+} \mathbf{n}^{+}+\boldsymbol{\tau}^{-} \mathbf{n}^{-}
\end{gathered}
$$

If $\mathbf{x}$ is on an edge/face $e$ lying on the boundary $\partial \Omega$, the above terms are defined by

$$
\begin{aligned}
& \{p\}=p, \quad[p]=p \mathbf{n}, \\
& \{\mathbf{v}\}=\mathbf{v}, \quad[\mathbf{v}]=\mathbf{v} \cdot \mathbf{n}, \\
& \{\boldsymbol{\tau}\}=\boldsymbol{\tau}, \quad[\boldsymbol{\tau}]=\boldsymbol{\tau} \mathbf{n},
\end{aligned}
$$


where $\mathbf{n}$ is the unit outward normal vector on $\partial \Omega$. We define a matrix-valued jump $\llbracket \cdot \rrbracket$ of a vector $\mathbf{v}$ as follows:

$$
\begin{gathered}
\llbracket \mathbf{v} \rrbracket=\frac{1}{2}\left(\mathbf{v}^{+} \otimes \mathbf{n}^{+}+\mathbf{n}^{+} \otimes \mathbf{v}^{+}+\mathbf{v}^{-} \otimes \mathbf{n}^{-}+\mathbf{n}^{-} \otimes \mathbf{v}^{-}\right), \quad \text { if } \mathbf{x} \in e \in \mathcal{E}_{h^{\prime}}^{i} \\
\llbracket \mathbf{v} \rrbracket=\frac{1}{2}(\mathbf{v} \otimes \mathbf{n}+\mathbf{n} \otimes \mathbf{v}), \quad \text { if } \mathbf{x} \in e \in \mathcal{E}_{h} \cap \partial \Omega,
\end{gathered}
$$

where $\mathbf{v} \otimes \mathbf{n}$ denotes the matrix whose $(i, j)$ th component is $v_{i} n_{j}$ for two vectors $\mathbf{v}$ and $\mathbf{n}$.

Now, we are ready to introduce a framework to derive DG methods for problem (2.1). Following [4, 7], we first derive a variational formulation for problem (2.1). Taking a double dot product with a matrix-valued function $\boldsymbol{\tau}$ on both sides of the first equation of (2.1) and integrating by parts over $K$, we have

$$
\int_{K} \mathcal{A} \boldsymbol{\sigma}: \boldsymbol{\tau} d x=-\int_{K} \mathbf{u} \cdot(\nabla \cdot \boldsymbol{\tau}) d x+\int_{\partial K} \mathbf{u} \cdot(\boldsymbol{\tau} \mathbf{n}) d s
$$

Multiplying the second equation of (2.1) by a vector-valued function $\mathbf{v}$ and integrating by parts over $K$ yields

$$
\int_{K} \boldsymbol{\sigma}: \boldsymbol{\varepsilon}(\mathbf{v}) d x=\int_{\partial K} \mathbf{v} \cdot(\boldsymbol{\sigma} \mathbf{n}) d s+\int_{K} \mathbf{f} \cdot \mathbf{v} d x
$$

Motivated by the above two identities, we may define our DG method as follows. Find an approximate solution $\left(\boldsymbol{\sigma}_{h}, \mathbf{u}_{h}\right) \in \Sigma_{h} \times \mathbf{V}_{h}$ such that

$$
\begin{gathered}
\int_{K} \mathcal{A} \boldsymbol{\sigma}_{h}: \boldsymbol{\tau} d x=-\int_{K} \mathbf{u}_{h} \cdot(\nabla \cdot \boldsymbol{\tau}) d x+\int_{\partial K} \widehat{\mathbf{u}}_{h} \cdot(\boldsymbol{\tau} \mathbf{n}) d s, \\
\int_{K} \boldsymbol{\sigma}_{h}: \boldsymbol{\varepsilon}(\mathbf{v}) d x=\int_{\partial K} \mathbf{v} \cdot\left(\widehat{\boldsymbol{\sigma}}_{h} \mathbf{n}\right) d s+\int_{K} \mathbf{f} \cdot \mathbf{v} d x
\end{gathered}
$$

for all $(\boldsymbol{\tau}, \mathbf{v}) \in \boldsymbol{\Sigma}_{h} \times \mathbf{V}_{h}$ and all $K \in \boldsymbol{\tau}_{h}$. Note that any function with the hat superscript is only defined over all faces of the triangulation $\tau_{h}$, which is called a numerical trace. Since $\sigma_{h}$ is symmetric, it is natural to choose $\widehat{\sigma}_{h}$ as a symmetric matrix-valued function. Moreover, we only consider the case where the numerical traces are single valued over all faces (conservation).

\subsection{Numerical Traces and the LDG Method}

We begin by producing a stability identity for the continuous problem (2.1), a crucial step in constructing feasible numerical traces to get a stable DG method from (2.12)-(2.13). For this, taking a double dot product with $\sigma$ on both sides of the first equation of (2.1) and then integrating over $\Omega$, we have

$$
\int_{\Omega} \mathcal{A} \boldsymbol{\sigma}: \boldsymbol{\sigma} d x-\int_{\Omega} \varepsilon(\mathbf{u}): \boldsymbol{\sigma} d x=0
$$


Multiplying the second equation of (2.1) by $\mathbf{u}$ and then integrating over $\Omega$ again, we find from the homogeneous boundary condition of $\mathbf{u}$ that

$$
\int_{\Omega} \varepsilon(\mathbf{u}): \boldsymbol{\sigma} d x=\int_{\Omega} \mathbf{f} \cdot \mathbf{u} d x
$$

Now adding these two equations gives

$$
\int_{\Omega} A \boldsymbol{\sigma}: \boldsymbol{\sigma} d x=\int_{\Omega} \mathbf{f} \cdot \mathbf{u} d x
$$

This is the stability identity corresponding to the continuous problem (2.1).

Next, we mimic the above derivation to get a discrete analogue of the stability identity (2.16) for the DG method (2.12)-(2.13). Taking $\boldsymbol{\tau}=\boldsymbol{\sigma}_{h}$ in (2.12) and summing over all $K \in \tau_{h}$, we have

$$
\int_{\Omega} \mathcal{A} \boldsymbol{\sigma}_{h}: \boldsymbol{\sigma}_{h} d x-\sum_{K \in \tau_{h}}\left(-\int_{K} \mathbf{u}_{h} \cdot\left(\nabla \cdot \boldsymbol{\sigma}_{h}\right) d x+\int_{\partial K} \widehat{\mathbf{u}}_{h} \cdot\left(\boldsymbol{\sigma}_{h} \mathbf{n}\right) d s\right)=0
$$

Similarly, summing up (2.13) over all $K \in \tau_{h}$ with $\mathbf{v}=\mathbf{u}_{h}$, we come to

$$
\sum_{K \in \tau_{h}}\left(\int_{K} \boldsymbol{\sigma}_{h}: \boldsymbol{\varepsilon}\left(\mathbf{u}_{h}\right) d x-\int_{\partial K} \mathbf{u}_{h} \cdot\left(\widehat{\boldsymbol{\sigma}}_{h} \mathbf{n}\right) d s\right)=\int_{\Omega} \mathbf{f} \cdot \mathbf{u}_{h} d x
$$

Adding the last two equations gives

$$
\int_{\Omega} A \sigma_{h}: \sigma_{h} d x+\Theta_{h}=\int_{\Omega} \mathbf{f} \cdot \mathbf{u}_{h} d x
$$

where

$$
\Theta_{h}:=\sum_{K \in \mathcal{Z}_{h}} \int_{K}\left(\mathbf{u}_{h} \cdot\left(\nabla \cdot \boldsymbol{\sigma}_{h}\right)+\boldsymbol{\sigma}_{h}: \boldsymbol{\varepsilon}\left(\mathbf{u}_{h}\right)\right) d x-\sum_{K \in \mathcal{T}_{h}} \int_{\partial K}\left(\widehat{\mathbf{u}}_{h} \cdot\left(\boldsymbol{\sigma}_{h} \mathbf{n}\right)-\mathbf{u}_{h} \cdot\left(\widehat{\boldsymbol{\sigma}}_{h} \mathbf{n}\right)\right) d s .
$$

Lemma 2.1. Assume $\boldsymbol{\sigma}_{h}$ and $\widehat{\boldsymbol{\sigma}}_{h}$ are both symmetric, and the numerical traces $\widehat{\boldsymbol{\sigma}}_{h}$ and $\widehat{\mathbf{u}}_{h}$ are single valued over all $e \in \mathcal{E}_{h}$. Then

$$
\begin{gathered}
\sum_{K \in \tau_{h}} \int_{\partial K \backslash \partial \Omega} \mathbf{u}_{h} \cdot\left(\boldsymbol{\sigma}_{h} \mathbf{n}\right) d s=\sum_{e \in \mathcal{E}_{h}^{i}} \int_{e}\left[\boldsymbol{\sigma}_{h}\right] \cdot\left\{\mathbf{u}_{h}\right\}+\left\{\boldsymbol{\sigma}_{h}\right\}: \llbracket \mathbf{u}_{h} \rrbracket d s, \\
\sum_{K \in \tau_{h}} \int_{\partial K \backslash \partial \Omega} \widehat{\mathbf{u}}_{h} \cdot\left(\boldsymbol{\sigma}_{h} \mathbf{n}\right) d s=\sum_{e \in \mathcal{E}_{h}^{i}} \int_{e}\left[\boldsymbol{\sigma}_{h}\right] \cdot \widehat{\mathbf{u}}_{h} d s, \\
\sum_{K \in \tau_{h}} \int_{\partial K \backslash \partial \Omega} \mathbf{u}_{h} \cdot\left(\widehat{\boldsymbol{\sigma}}_{h} \mathbf{n}\right) d s=\sum_{e \in \mathcal{E}_{h}^{i}} \int_{e} \widehat{\boldsymbol{\sigma}}_{h}: \llbracket \mathbf{u}_{h} \rrbracket d s .
\end{gathered}
$$


Proof. We only prove the first identity, and the other two ones can be obtained in the similar manners. The left-hand side of (2.21) can be rewritten as

$$
\sum_{K \in \tau_{h}} \int_{\partial K \backslash \partial \Omega} \mathbf{u}_{h} \cdot\left(\boldsymbol{\sigma}_{h} \mathbf{n}\right) d s=\sum_{e \in \mathcal{E}_{h}^{i}} \int_{e}\left(\mathbf{u}_{h}^{+} \cdot\left(\boldsymbol{\sigma}_{h}^{+} \mathbf{n}^{+}\right)+\mathbf{u}_{h}^{-} \cdot\left(\boldsymbol{\sigma}_{h}^{-} \mathbf{n}^{-}\right)\right) d s .
$$

On the other hand,

$$
\begin{aligned}
& \sum_{e \in \mathcal{E}_{h}^{i}} \int_{e}\left[\boldsymbol{\sigma}_{h}\right] \cdot\left\{\mathbf{u}_{h}\right\} d s=\sum_{e \in \mathcal{E}_{h}^{i}} \int_{e} \frac{1}{2}\left(\mathbf{u}_{h}^{+} \cdot\left(\boldsymbol{\sigma}_{h}^{+} \mathbf{n}^{+}\right)+\mathbf{u}_{h}^{+} \cdot\left(\boldsymbol{\sigma}_{h}^{-} \mathbf{n}^{-}\right)+\mathbf{u}_{h}^{-} \cdot\left(\boldsymbol{\sigma}_{h}^{+} \mathbf{n}^{+}\right)+\mathbf{u}_{h}^{-} \cdot\left(\boldsymbol{\sigma}_{h}^{-} \mathbf{n}^{-}\right)\right) d s, \\
& \sum_{e \in \mathcal{E}_{h}^{i}} \int_{e}\left\{\boldsymbol{\sigma}_{h}\right\}: \llbracket \mathbf{u}_{h} \rrbracket d s=\sum_{e \in \mathcal{E}_{h}^{i}} \int_{e} \frac{1}{2}\left(\mathbf{n}^{+} \cdot\left(\boldsymbol{\sigma}_{h}^{+} \mathbf{u}_{h}^{+}\right)+\mathbf{n}^{-} \cdot\left(\boldsymbol{\sigma}_{h}^{+} \mathbf{u}_{h}^{-}\right)+\mathbf{n}^{+} \cdot\left(\boldsymbol{\sigma}_{h}^{-} \mathbf{u}_{h}^{+}\right)+\mathbf{n}^{-} \cdot\left(\boldsymbol{\sigma}_{h}^{-} \mathbf{u}_{h}^{-}\right)\right) d s .
\end{aligned}
$$

Hence, the identity (2.21) is a direct consequence of (2.24)-(2.25).

By Lemma 2.1 and using integration by parts twice, we see that

$$
\begin{aligned}
\Theta_{h}= & \sum_{K \in \tau_{h}}\left(\int_{K} \nabla \cdot\left(\boldsymbol{\sigma}_{h} \mathbf{u}_{h}\right) d x-\int_{\partial K} \widehat{\mathbf{u}}_{h} \cdot\left(\boldsymbol{\sigma}_{h} \mathbf{n}\right) d s-\int_{\partial K} \mathbf{u}_{h} \cdot\left(\widehat{\boldsymbol{\sigma}}_{h} \mathbf{n}\right) d s\right) \\
= & \sum_{K \in \tau_{h}}\left(\int_{\partial K} \mathbf{n} \cdot\left(\boldsymbol{\sigma}_{h} \mathbf{u}_{h}\right) d s-\int_{\partial K} \widehat{\mathbf{u}}_{h} \cdot\left(\boldsymbol{\sigma}_{h} \mathbf{n}\right) d s-\int_{\partial K} \mathbf{u}_{h} \cdot\left(\widehat{\boldsymbol{\sigma}}_{h} \mathbf{n}\right) d s\right) \\
= & \sum_{e \in \mathcal{E}_{h}^{i}} \int_{e}\left(\left[\boldsymbol{\sigma}_{h}\right] \cdot\left(\left\{\mathbf{u}_{h}\right\}-\widehat{\mathbf{u}}_{h}\right)+\left(\left\{\boldsymbol{\sigma}_{h}\right\}-\widehat{\boldsymbol{\sigma}}_{h}\right): \llbracket \mathbf{u}_{h} \rrbracket\right) d s \\
& +\int_{\partial \Omega}\left(\mathbf{u}_{h} \cdot\left(\left(\boldsymbol{\sigma}_{h}-\widehat{\boldsymbol{\sigma}}_{h}\right) \mathbf{n}\right)-\widehat{\mathbf{u}}_{h} \cdot\left(\boldsymbol{\sigma}_{h} \mathbf{n}\right)\right) d s .
\end{aligned}
$$

Thus, if $e \in \mathcal{E}_{h^{\prime}}^{i}$ we take

$$
\begin{aligned}
& \widehat{\boldsymbol{\sigma}}_{h}=\left\{\boldsymbol{\sigma}_{h}\right\}-C_{11} \llbracket \mathbf{u}_{h} \rrbracket, \\
& \widehat{\mathbf{u}}_{h}=\left\{\mathbf{u}_{h}\right\}-C_{22}\left[\boldsymbol{\sigma}_{h}\right],
\end{aligned}
$$

and if $e \in \mathcal{\varepsilon}_{h} \cap \partial \Omega$, we take

$$
\begin{gathered}
\widehat{\boldsymbol{\sigma}}_{h}=\boldsymbol{\sigma}_{h}-C_{11} \llbracket \mathbf{u}_{h} \rrbracket, \\
\widehat{\mathbf{u}}_{h}=0,
\end{gathered}
$$

where $C_{11}$ and $C_{22}$ are two nonnegative continuous functions on $e$. When $C_{22}=0$, the corresponding method is called the LDG method for linear elasticity, viewed as a generalization of the LDG method for second-order elliptic problems in [4, 7]. 
For the above choice of numerical traces, we have by some direct manipulation that

$$
\Theta_{h}=\sum_{e \in \mathcal{E}_{h}^{i}} \int_{e}\left(C_{22}\left[\boldsymbol{\sigma}_{h}\right]^{2}+C_{11} \llbracket \mathbf{u}_{h} \rrbracket^{2}\right) d s+\int_{\partial \Omega} C_{11} \llbracket \mathbf{u}_{h} \rrbracket^{2} d s \geq 0
$$

Then, the combination of (2.19) and (2.29) allows a discrete stability estimate:

$$
\left\|\boldsymbol{\sigma}_{h}\right\|_{0}^{2} \lesssim \int_{\Omega} \mathbf{f} \cdot \mathbf{u}_{h} d x
$$

which is essential in constructing a reliable DG method (cf. [7]).

Let us further show the unique solvability of problem (2.12)-(2.13) with the numerical traces given by (2.27) and (2.28), whenever $C_{11}>0$ and the finite element spaces $\Sigma_{h}$ and $\mathbf{V}_{h}$ satisfy condition (2.5). In fact, it suffices to verify that this DG method only has zero solution when $\mathbf{f}=\mathbf{0}$. By setting $\mathbf{f}=\mathbf{0}$, the stability identity (2.19) gives

$$
\int_{\Omega} A \sigma_{h}: \sigma_{h} d x+\sum_{e \in \mathcal{E}_{h}^{i}} \int_{e}\left(C_{22}\left[\sigma_{h}\right]^{2}+C_{11} \llbracket \mathbf{u}_{h} \rrbracket^{2}\right) d s d s+\int_{\partial \Omega} C_{11} \llbracket \mathbf{u}_{h} \rrbracket^{2} d s=0,
$$

which implies that $\boldsymbol{\sigma}_{h}=\mathbf{0}$ in $\Omega, \llbracket \mathbf{u}_{h} \rrbracket=0$ on $\mathcal{E}_{h^{\prime}}^{i}$ and $\mathbf{u}_{h}=\mathbf{0}$ on $\partial \Omega$, provided that $C_{11}>0$. Therefore, from (2.12) the definition of $\widehat{\mathbf{u}}_{h}$ (cf. (2.27) and (2.28)), and Lemma 2.1, we know that

$$
\int_{K} \boldsymbol{\tau}: \varepsilon\left(\mathbf{u}_{h}\right) d x=0, \quad \forall \boldsymbol{\tau} \in \Sigma_{h}
$$

which, due to (2.5), implies that $\boldsymbol{\varepsilon}\left(\mathbf{u}_{h}\right)=\mathbf{0}$. By Korn's inequality (2.34) on the discontinuous finite element space, given below, it is easy to see that $\left\|\mathbf{u}_{h}\right\|_{1, h}=0$. Then with $\mathbf{u}_{h}=\mathbf{0}$ on $\partial \Omega$ and $\llbracket \mathbf{u}_{h} \rrbracket=0$, we conclude that $\mathbf{u}_{h}=\mathbf{0}$ in $\Omega$, as required.

Remark 2.2. The standard Korn's inequality states that (cf. [25])

$$
\|\mathbf{v}\|_{1}^{2} \lesssim\|\varepsilon(\mathbf{v})\|_{0}^{2}+\int_{\partial \Omega}|\mathbf{v}|^{2} d s, \quad \forall \mathbf{v} \in H^{1}(\Omega)^{d}
$$

The following Korn's inequality on the discontinuous finite-element space is given in [26]:

$$
\|\mathbf{v}\|_{1, h}^{2} \lesssim\|\varepsilon(\mathbf{v})\|_{0, h}^{2}+\sum_{e \in \varepsilon_{h}} h_{e}^{-1}\|\llbracket \mathbf{v} \rrbracket\|_{0, e^{\prime}}^{2} \quad \forall \mathbf{v} \in \mathbf{V}_{h}
$$

As in [4, 7], the DG method (2.12)-(2.13) with the numerical traces (2.27)-(2.28) can also be written in a mixed formulation (cf. [27]). After some direct manipulation, the 
approximate solution $\left(\boldsymbol{\sigma}_{h}, \mathbf{u}_{h}\right)$ can be characterized as the unique solution of the following variational problem. Find $\left(\sigma_{h}, \mathbf{u}_{h}\right) \in \Sigma_{h} \times \mathbf{V}_{h}$ such that

$$
\begin{gathered}
a\left(\boldsymbol{\sigma}_{h}, \boldsymbol{\tau}\right)+b\left(\mathbf{u}_{h}, \boldsymbol{\tau}\right)=0, \\
-b\left(\mathbf{v}, \boldsymbol{\sigma}_{h}\right)+c\left(\mathbf{u}_{h}, \mathbf{v}\right)=F(\mathbf{v}),
\end{gathered}
$$

for all $(\boldsymbol{\tau}, \mathbf{v}) \in \boldsymbol{\Sigma}_{h} \times \mathbf{V}_{h}$, where

$$
\begin{gathered}
a(\boldsymbol{\sigma}, \boldsymbol{\tau}):=\int_{\Omega} \mathcal{A} \boldsymbol{\sigma}: \boldsymbol{\tau} d x+\int_{\mathcal{E}_{h}^{i}} C_{22}[\boldsymbol{\sigma}] \cdot[\boldsymbol{\tau}] d s, \\
b(\mathbf{v}, \boldsymbol{\tau}):=\sum_{K \in \mathcal{\tau}_{h}} \int_{K} \mathbf{v} \cdot(\nabla \cdot \boldsymbol{\tau}) d x-\int_{\mathcal{E}_{h}^{i}}\{\mathbf{v}\} \cdot[\boldsymbol{\tau}] d s, \\
\mathcal{c}(\mathbf{u}, \mathbf{v}):=\int_{\mathcal{E}_{h}} C_{11} \llbracket \mathbf{u} \rrbracket: \llbracket \mathbf{v} \rrbracket d s, \\
F(\mathbf{v}):=\int_{\Omega} \mathbf{f} \cdot \mathbf{v} d x .
\end{gathered}
$$

Remark 2.3. If we take $C_{11}=0$ and $C_{22}=\alpha h_{e}^{s}$, the mixed formulation (2.35)-(2.36) will be reduced to the one adopted in [20].

\section{Error Analysis for the LDG Method}

In this section, we provide an error analysis for the DG method (2.35)-(2.36) in the case $C_{22}=$ 0 , which is named as the LDG method in the last section. For this purpose, we are going to derive a primal formulation for the approximate method. First we introduce a global lifting operator $\mathbf{r}:\left(L^{2}\left(\varepsilon_{h}\right)\right)_{d \times d}^{s} \rightarrow \Sigma_{h}$ defined by

$$
\int_{\Omega} \mathbf{r}(\boldsymbol{\phi}): \boldsymbol{\tau} d x=-\int_{\varepsilon_{h}} \boldsymbol{\phi}:\{\boldsymbol{\tau}\} d s, \quad \forall \boldsymbol{\tau} \in \boldsymbol{\Sigma}_{h}, \boldsymbol{\phi} \in\left(L^{2}\left(\mathcal{\varepsilon}_{h}\right)\right)_{d \times d}^{s}
$$

Moreover, for each $e \in \mathcal{E}_{h}$, we introduce a local lifting operator $\mathbf{r}_{e}:\left(L^{2}(e)\right)_{d \times d}^{s} \rightarrow \Sigma_{h}$ defined by

$$
\int_{\Omega} \mathbf{r}_{e}(\phi): \boldsymbol{\tau} d x=-\int_{e} \boldsymbol{\phi}:\{\boldsymbol{\tau}\} d s, \quad \forall \boldsymbol{\tau} \in \boldsymbol{\Sigma}_{h}, \boldsymbol{\phi} \in\left(L^{2}(e)\right)_{d \times d}^{s}
$$

Then it is easy to check that $\mathbf{r}_{e}(\boldsymbol{\phi})$ is only nonzero in the triangles with $e$ as one edge, and there holds the identity

$$
\mathbf{r}(\boldsymbol{\phi})=\sum_{e \in \mathcal{E}_{h}} \mathbf{r}_{e}\left(\left.\boldsymbol{\phi}\right|_{e}\right), \quad \forall \boldsymbol{\phi} \in\left(L^{2}\left(\mathcal{\varepsilon}_{h}\right)\right)_{d \times d}^{s}
$$


Now we express $\boldsymbol{\sigma}_{h}$ in terms of $\mathbf{u}_{h}$. From the first equation in Lemma 2.1 and (2.35), it follows that

$$
\begin{aligned}
\int_{\Omega} \mathcal{A} \boldsymbol{\sigma}_{h}: \boldsymbol{\tau} d x & =-\sum_{K \in \mathcal{T}_{h}} \int_{K} \mathbf{u}_{h} \cdot(\nabla \cdot \boldsymbol{\tau}) d x+\int_{\mathcal{E}_{h}^{i}}\left\{\mathbf{u}_{h}\right\} \cdot[\boldsymbol{\tau}] d s \\
& =\sum_{K \in \mathcal{\tau}_{h}} \int_{K} \boldsymbol{\varepsilon}\left(\mathbf{u}_{h}\right): \boldsymbol{\tau} d x-\int_{\mathcal{E}_{h}} \llbracket \mathbf{u}_{h} \rrbracket:\{\boldsymbol{\tau}\} d s \\
& =\sum_{K \in \mathcal{Z}_{h}} \int_{K} \varepsilon\left(\mathbf{u}_{h}\right): \boldsymbol{\tau} d x+\int_{\Omega} \mathbf{r}\left(\llbracket \mathbf{u}_{h} \rrbracket\right): \boldsymbol{\tau} d x
\end{aligned}
$$

for all $\boldsymbol{\tau} \in \Sigma_{h}$. Then by (2.5), we get

$$
\mathcal{A} \sigma_{h}=\varepsilon\left(\mathbf{u}_{h}\right)+\mathbf{r}\left(\llbracket \mathbf{u}_{h} \rrbracket\right)
$$

that is,

$$
\boldsymbol{\sigma}_{h}=2 \mu\left(\varepsilon\left(\mathbf{u}_{h}\right)+\mathbf{r}\left(\llbracket \mathbf{u}_{h} \rrbracket\right)\right)+\lambda \operatorname{tr}\left(\varepsilon\left(\mathbf{u}_{h}\right)+\mathbf{r}\left(\llbracket \mathbf{u}_{h} \rrbracket\right)\right) \delta .
$$

Substituting $\sigma_{h}$ from the last equation into (2.36), we get a primal formulation for the LDG method as follows. Find $\mathbf{u}_{h} \in \mathbf{V}_{h}$ such that

$$
a_{h}\left(\mathbf{u}_{h}, \mathbf{v}\right)=\int_{\Omega} \mathbf{f} \cdot \mathbf{v} d x, \quad \forall \mathbf{v} \in \mathbf{V}_{h}
$$

where

$$
\begin{aligned}
a_{h}(\mathbf{w}, \mathbf{v}):= & \sum_{K \in \tau_{h}} \int_{K} 2 \mu(\varepsilon(\mathbf{w})+\mathbf{r}(\llbracket \mathbf{w} \rrbracket)):(\varepsilon(\mathbf{v})+\mathbf{r}(\llbracket \mathbf{v} \rrbracket)) d x \\
& +\sum_{K \in \tau_{h}} \int_{K} \lambda \operatorname{tr}(\varepsilon(\mathbf{w})+\mathbf{r}(\llbracket \mathbf{w} \rrbracket)) \operatorname{tr}(\varepsilon(\mathbf{v})+\mathbf{r}(\llbracket \mathbf{v} \rrbracket)) d x+\int_{\varepsilon_{h}} C_{11} \llbracket \mathbf{w} \rrbracket: \llbracket \mathbf{v} \rrbracket d s .
\end{aligned}
$$

Next, we consider the consistency of the method (3.7). Assume that $(\boldsymbol{\sigma}, \mathbf{u}) \in$ $\left(H^{s+1}(\Omega)\right)_{d \times d}^{s} \times H^{s+2}(\Omega)^{d}$, where $s \geq 0$ is some nonnegative integer, and $\mathbf{u}$ is the exact solution of (2.1). It is easy to see that $\llbracket \mathbf{u} \rrbracket=0$ on $\boldsymbol{\varepsilon}_{h}$. Therefore, for any $\mathbf{v} \in \mathbf{V}_{h}$, we have

$$
\begin{aligned}
a_{h}(\mathbf{u}, \mathbf{v}) & =\sum_{K \in \tau_{h}} \int_{K}(2 \mu \varepsilon(\mathbf{u}):(\varepsilon(\mathbf{v})+\mathbf{r}(\llbracket \mathbf{v} \rrbracket))+\lambda \operatorname{tr}(\varepsilon(\mathbf{u})) \operatorname{tr}(\varepsilon(\mathbf{v})+\mathbf{r}(\llbracket \mathbf{v} \rrbracket))) d x \\
& =\sum_{K \in \tau_{h}} \int_{K} \sigma:(\varepsilon(\mathbf{v})+\mathbf{r}(\llbracket \mathbf{v} \rrbracket)) d x .
\end{aligned}
$$


From (3.9), the definition of lifting operator $\mathbf{r}$ (see (3.1)), and the fact that $\{\boldsymbol{\sigma}\}=\boldsymbol{\sigma}$, we further find that

$$
a_{h}(\mathbf{u}, \mathbf{v})=\tilde{a}(\mathbf{u}, \mathbf{v})+\int_{\varepsilon_{h}} \llbracket \mathbf{v} \rrbracket:\left\{\boldsymbol{\sigma}-\mathbf{P}_{h} \boldsymbol{\sigma}\right\} d s+\sum_{K \in \tau_{h}} \int_{K}\left(\boldsymbol{\sigma}-\mathbf{P}_{h} \boldsymbol{\sigma}\right): \mathbf{r}(\llbracket \mathbf{v} \rrbracket) d x,
$$

where

$$
\tilde{a}(\mathbf{u}, \mathbf{v}):=\sum_{K \in \mathcal{\tau}_{h}} \int_{K} \boldsymbol{\sigma}: \boldsymbol{\varepsilon}(\mathbf{v}) d x-\int_{\varepsilon_{h}} \llbracket \mathbf{v} \rrbracket: \sigma d s .
$$

Noting that $[\sigma]=0$ in $\mathcal{E}_{h}^{i}$ and using integration by parts and the same technique for deriving (2.21), we deduce from (2.1) that

$$
\tilde{a}_{h}(\mathbf{u}, \mathbf{v})=-\sum_{K \in \tau_{h}} \int_{K} \nabla \cdot \boldsymbol{\sigma} d x=\int_{\Omega} \mathbf{f} \cdot \mathbf{v} d x
$$

Hence, we know that the LDG method (3.7) is not consistent with respect to the bilinear form $a_{h}(\cdot, \cdot)$, but it admits the following identity:

$$
a_{h}\left(\mathbf{u}-\mathbf{u}_{h}, \mathbf{v}\right)=\int_{\varepsilon_{h}} \llbracket \mathbf{v} \rrbracket:\left\{\boldsymbol{\sigma}-\mathbf{P}_{h} \boldsymbol{\sigma}\right\} d s+\sum_{K \in \tau_{h}} \int_{K}\left(\boldsymbol{\sigma}-\mathbf{P}_{h} \boldsymbol{\sigma}\right): \mathbf{r}(\llbracket \mathbf{v} \rrbracket) d x, \quad \forall \mathbf{v} \in \mathbf{V}_{h}
$$

It is worth noting that the LDG method does not contain any parameter which needs to be quantified a priori. In what follows, we choose $C_{11}=\eta_{e} h_{e}^{-1}$ on each $e \in \mathcal{E}_{h}$ with $\left\{\eta_{e}\right\}_{e \in \varepsilon_{h}}$ having a uniform positive bound from above and below.

Next, we present a useful estimate for the local lifting operator $\mathbf{r}_{e}$. For this, let $\mathbf{V}(h):=$ $\mathbf{V}_{h}+H_{0}^{1}(\Omega)^{d}$ and define the mesh-dependent energy norm for $\mathbf{v} \in \mathbf{V}(h)$ by

$$
\left.\|\mathbf{v}\|\right|^{2}=\|\varepsilon(\mathbf{v})\|_{0, h}^{2}+\sum_{e \in \varepsilon_{h}} h_{e}^{-1}\|\llbracket \mathbf{v} \rrbracket\|_{0, e}^{2}
$$

Lemma 3.1. For any $\mathbf{v} \in \mathbf{V}(h)$ and $e \in \mathcal{E}_{h}$, one has

$$
\left\|\mathbf{r}_{e}(\llbracket \mathbf{v} \rrbracket)\right\|_{0, h}^{2} \lesssim h_{e}^{-1}\|\llbracket \mathbf{v} \rrbracket\|_{0, e}^{2}
$$

Proof. Since $\mathbf{v} \in H_{0}^{1}(\Omega)^{d}$ implies that $\llbracket \mathbf{v} \rrbracket=0$, it suffices to verify the result for $\mathbf{v} \in \mathbf{V}_{h}$. Taking $\boldsymbol{\phi}=\llbracket \mathbf{v} \rrbracket$ and $\boldsymbol{\tau}=\mathbf{r}_{e}(\llbracket \mathbf{v} \rrbracket)$ in (3.2), we get

$$
\left\|\mathbf{r}_{e}(\llbracket \mathbf{v} \rrbracket)\right\|_{0, h}^{2}=-\int_{e} \llbracket \mathbf{v} \rrbracket:\left\{\mathbf{r}_{e}(\llbracket \mathbf{v} \rrbracket)\right\} d s \leq\|\llbracket \mathbf{v} \rrbracket\|_{0, e}\left\|\left\{\mathbf{r}_{e}(\llbracket \mathbf{v} \rrbracket)\right\}\right\|_{0, e}
$$


On the other hand, by the scaling arguments, the trace theorem, and the inverse inequality,

$$
\left\|\left\{\mathbf{r}_{e}(\llbracket \mathbf{v} \rrbracket)\right\}\right\|_{0, e}^{2} \lesssim h_{e}^{-1}\left\|\mathbf{r}_{e}(\llbracket \mathbf{v} \rrbracket)\right\|_{0, h}^{2}+h_{e}\left|\mathbf{r}_{e}(\llbracket \mathbf{v} \rrbracket)\right|_{1, h}^{2} \lesssim h_{e}^{-1}\left\|\mathbf{r}_{e}(\llbracket \mathbf{v} \rrbracket)\right\|_{0, h}^{2}
$$

Therefore, (3.15) is a direct consequence of (3.16) and (3.17).

Lemma 3.2 (boundedness). For any $(\mathbf{w}, \mathbf{v}) \in \mathbf{V}(h) \times \mathbf{V}(h)$, it holds that

$$
a_{h}(\mathbf{w}, \mathbf{v}) \lesssim|\|\mathbf{w}\|||||\mathbf{v} \||
$$

Proof. According to the Cauchy-Schwarz inequality and the basic properties of $\mathbf{r}$ and $\mathbf{r}_{e}$, we have

$$
\begin{aligned}
& a_{h}(\mathbf{w}, \mathbf{v}) \\
& \lesssim \sum_{K \in \mathcal{Z}_{h}}\left(\int_{K}|\varepsilon(\mathbf{w})+\mathbf{r}(\llbracket \mathbf{w} \rrbracket)|^{2} d x \cdot \int_{K}|\varepsilon(\mathbf{v})+\mathbf{r}(\llbracket \mathbf{v} \rrbracket)|^{2} d x\right)^{1 / 2}+\int_{\varepsilon_{h}} \eta_{e} h_{e}^{-1} \llbracket \mathbf{w} \rrbracket: \llbracket \mathbf{v} \rrbracket d s \\
& \quad \lesssim\left(\|\varepsilon(\mathbf{w})\|_{0, h}^{2}+\|\mathbf{r}(\llbracket \mathbf{w} \rrbracket)\|_{0, h}^{2}\right)^{1 / 2} \cdot\left(\|\varepsilon(\mathbf{v})\|_{0, h}^{2}+\|\mathbf{r}(\llbracket \mathbf{v} \rrbracket)\|_{0, h}^{2}\right)^{1 / 2}+\int_{\varepsilon_{h}} \eta_{e} h_{e}^{-1} \llbracket \mathbf{w} \rrbracket: \llbracket \mathbf{v} \rrbracket d s \\
& \quad \lesssim\left(\|\varepsilon(\mathbf{w})\|_{0, h}^{2}+\sum_{e \in \varepsilon_{h}}\left\|\mathbf{r}_{e}(\llbracket \mathbf{w} \rrbracket)\right\|_{0, h}^{2}\right)^{1 / 2}\left(\|\varepsilon(\mathbf{v})\|_{0, h}^{2}+\sum_{e \in \varepsilon_{h}}\left\|\mathbf{r}_{e}(\llbracket \mathbf{v} \rrbracket)\right\|_{0, h}^{2}\right)^{1 / 2}+\int_{\varepsilon_{h}} \eta_{e} h_{e}^{-1} \llbracket \mathbf{w} \rrbracket: \llbracket \mathbf{v} \rrbracket d s,
\end{aligned}
$$

which, together with (3.15), yields

$$
a_{h}(\mathbf{w}, \mathbf{v}) \lesssim|\|\mathbf{w}\|||| \mathbf{v}\left\|\left|+\int_{\varepsilon_{h}} \eta_{e} h_{e}^{-1} \llbracket \mathbf{w} \rrbracket: \llbracket \mathbf{v} \rrbracket d s \lesssim\right|\right\| \mathbf{w}\||||\mathbf{v} \||,
$$

as required.

Lemma 3.3 (stability). For any $\mathbf{v} \in \mathbf{V}_{h}$, it holds that

$$
a_{h}(\mathbf{v}, \mathbf{v}) \gtrsim|\|\mathbf{v}\||^{2} .
$$


Proof. Using the Cauchy-Schwarz inequality and (3.15), we get

$$
\begin{aligned}
a_{h}(\mathbf{v}, \mathbf{v}) & \geq 2 \mu\|\varepsilon(\mathbf{v})+\mathbf{r}(\llbracket \mathbf{v} \rrbracket)\|_{0, h}^{2}+\int_{\varepsilon_{h}} \eta_{e} h_{e}^{-1}|\llbracket \mathbf{v} \rrbracket|^{2} d s \\
& \geq 2 \mu\|\varepsilon(\mathbf{v})\|_{0, h}^{2}+2 \mu\|\mathbf{r}(\llbracket \mathbf{v} \rrbracket)\|_{0, h}^{2}+4 \mu \sum_{K \in \tau_{h}} \int_{K} \varepsilon(\mathbf{v}): \mathbf{r}(\llbracket \mathbf{v} \rrbracket) d x+\eta_{0} \int_{\varepsilon_{h}} h_{e}^{-1}|\llbracket \mathbf{v} \rrbracket|^{2} d s \\
& \geq 2 \mu(1-\epsilon)\|\varepsilon(\mathbf{v})\|_{0, h}^{2}+2 \mu\left(1-\frac{1}{\epsilon}\right)\|\mathbf{r}(\llbracket \mathbf{v} \rrbracket)\|_{0, h}^{2}+\eta_{0} \int_{\varepsilon_{h}} h_{e}^{-1}|\llbracket \mathbf{v} \rrbracket|^{2} d s \\
& \geq 2 \mu(1-\epsilon)\|\varepsilon(\mathbf{v})\|_{0, h}^{2}+\left(2 \mu C_{1}+\eta_{0}-\frac{2 \mu C_{1}}{\epsilon}\right) \int_{\varepsilon_{h}} h_{e}^{-1}|\llbracket \mathbf{v} \rrbracket|^{2} d s .
\end{aligned}
$$

Here $\eta_{0}=\min _{e \in \varepsilon_{h}} \eta_{e}, 0<\epsilon<1$, and $C_{1}$ is the constant in (3.15). Therefore (3.21) is true if we choose $\epsilon$ such that $2 \mu C_{1}+\eta_{0}-2 \mu C_{1} / \epsilon>0$, that is, $2 \mu C_{1} /\left(2 \mu C_{1}+\eta_{0}\right)<\epsilon<1$.

Now, we are in a position to give error analysis for the LDG method (3.7). The main idea of our derivation is based on the framework on error analysis of DG methods for secondorder elliptic problems (cf. [5]). Let $\mathbf{Q}_{h}$ be an $L^{2}$-projection operator from $\mathbf{V}$ onto the finiteelement space $\mathbf{V}_{h}$. Let $\mathbf{P}_{h}$ denote the usual $L^{2}$-orthogonal projection operator onto $\boldsymbol{\Sigma}_{h}$. For simplicity, we still write $\mathbf{Q}_{h}$ (resp., $\mathbf{P}_{h}$ ) for $\left.\mathbf{Q}_{h}\right|_{K}$ (resp., $\left.\mathbf{P}_{h}\right|_{K}$ ). Using the standard scaling arguments (cf. [24]), we can easily obtain error estimates for the operators $\mathbf{Q}_{h}$ and $\mathbf{P}_{h}$.

Lemma 3.4. Let $\mathbf{v} \in H^{s+2}(K)^{d}, \boldsymbol{\sigma} \in\left(H^{s+1}(\Omega)\right)_{d \times d}^{s}$ with $s \geq 0$. Then for all $K \in \tau_{h}$, one has

$$
\begin{gathered}
\left\|\mathbf{v}-\mathbf{Q}_{h} \mathbf{v}\right\|_{0, K}+h_{K}\left|\mathbf{v}-\mathbf{Q}_{h} \mathbf{v}\right|_{1, K} \lesssim h_{K}^{\min \{s+1, k\}+1}\|\mathbf{v}\|_{s+2, K^{\prime}} \\
\left\|\mathbf{v}-\mathbf{Q}_{h} \mathbf{v}\right\|_{0, \partial K} \lesssim h_{K}^{\min \{s+1, k\}+1 / 2}\|\mathbf{v}\|_{s+2, K} \\
\left\|\boldsymbol{\sigma}-\mathbf{P}_{h} \boldsymbol{\sigma}\right\|_{0, K} \lesssim h_{K}^{\min \{s+1, l+1\}}\|\boldsymbol{\sigma}\|_{s+1, K} \\
h_{e}^{1 / 2}\left\|\boldsymbol{\sigma}-\mathbf{P}_{h} \boldsymbol{\sigma}\right\|_{0, e} \lesssim h_{K}^{\min \{s+1, l+1\}}\|\boldsymbol{\sigma}\|_{s+1, K} .
\end{gathered}
$$

Theorem 3.5. Let $(\boldsymbol{\sigma}, \mathbf{u}) \in\left(H^{s+1}(\Omega)\right)_{d \times d}^{s} \times H^{s+2}(\Omega)^{d}$, where s is a nonnegative integer and $\mathbf{u}$ is the solution of (2.1). Let $\mathbf{u}_{h} \in \mathbf{V}_{h}$ be the solution of (3.7). Then one has

$$
\left\|\left|\mathbf{u}-\mathbf{u}_{h}\right|\right\| \lesssim h^{\min \{s+1, k, l+1\}}\|\mathbf{u}\|_{s+2} .
$$


Proof. From the stability (3.21), the identity (3.13), the boundedness (3.18), and Lemmas 3.1 and 3.4, it follows that

$$
\begin{aligned}
\left.\left\|\mathbf{Q}_{h} \mathbf{u}-\mathbf{u}_{h}\right\|\right|^{2} \lesssim & a_{h}\left(\mathbf{Q}_{h} \mathbf{u}-\mathbf{u}_{h}, \mathbf{Q}_{h} \mathbf{u}-\mathbf{u}_{h}\right) \\
= & a_{h}\left(\mathbf{Q}_{h} \mathbf{u}-\mathbf{u}, \mathbf{Q}_{h} \mathbf{u}-\mathbf{u}_{h}\right)+a_{h}\left(\mathbf{u}-\mathbf{u}_{h}, \mathbf{Q}_{h} \mathbf{u}-\mathbf{u}_{h}\right) \\
= & a_{h}\left(\mathbf{Q}_{h} \mathbf{u}-\mathbf{u}, \mathbf{Q}_{h} \mathbf{u}-\mathbf{u}_{h}\right)+\int_{\varepsilon_{h}} \llbracket \mathbf{Q}_{h} \mathbf{u}-\mathbf{u}_{h} \rrbracket:\left\{\boldsymbol{\sigma}-\mathbf{P}_{h} \boldsymbol{\sigma}\right\} d s \\
& +\sum_{K \in \tau_{h}} \int_{K}\left(\boldsymbol{\sigma}-\mathbf{P}_{h} \boldsymbol{\sigma}\right): \mathbf{r}\left(\llbracket \mathbf{Q}_{h} \mathbf{u}-\mathbf{u}_{h} \rrbracket\right) d x \\
\lesssim & \left\|\mathbf{Q}_{h} \mathbf{u}-\mathbf{u}\left|\|\| \mathbf{Q}_{h} \mathbf{u}-\mathbf{u}_{h}\right|\right\|+h^{\min \{s+1, l+1\}}\|\mathbf{u}\|_{s+2}\left\|\left|\mathbf{Q}_{h} \mathbf{u}-\mathbf{u}_{h}\right|\right\|,
\end{aligned}
$$

that is,

$$
\left\|\left|\mathbf{Q}_{h} \mathbf{u}-\mathbf{u}_{h}\right|\right\| \lesssim\left\|\left|\mathbf{Q}_{h} \mathbf{u}-\mathbf{u}\right|\right\|+h^{\min \{s+1, l+1\}}\|\mathbf{u}\|_{s+2} .
$$

Therefore, using the triangle inequality and Lemma 3.4, we further have

$$
\begin{aligned}
\left\|\left|\mathbf{u}-\mathbf{u}_{h}\right|\right\| & \leq\left\|\left|\mathbf{u}-\mathbf{Q}_{h} \mathbf{u}\right|\right\|+\left\|\left|\mathbf{Q}_{h} \mathbf{u}-\mathbf{u}_{h}\right|\right\| \\
& \lesssim\left\|\mathbf{u}-\mathbf{Q}_{h} \mathbf{u} \mid\right\|+h^{\min \{s+1, l+1\}}\|\mathbf{u}\|_{s+2, \Omega} \\
& \lesssim h^{\min \{s+1, k, l+1\}}\|\mathbf{u}\|_{s+2, \Omega}
\end{aligned}
$$

Theorem 3.6. Let $(\boldsymbol{\sigma}, \mathbf{u}) \in\left(H^{s+1}(\Omega)\right)_{d \times d}^{s} \times H^{s+2}(\Omega)^{d}$, where s is a nonnegative integer and $\mathbf{u}$ is the solution of (2.1). Let $\mathbf{u}_{h} \in \mathbf{V}_{h}$ be the solution of (3.7). Then one has

$$
\left\|\mathbf{u}-\mathbf{u}_{h}\right\|_{1, h} \lesssim h^{\min \{s+1, k, l+1\}}\|\mathbf{u}\|_{s+2}
$$

Proof. By the triangle inequality and Korn's inequality over $\mathbf{V}_{h}$ (cf. (2.34)), we know that

$$
\begin{aligned}
\left\|\mathbf{u}-\mathbf{u}_{h}\right\|_{1, h} & \lesssim\left\|\mathbf{u}-\mathbf{Q}_{h} \mathbf{u}\right\|_{1, h}+\left\|\mathbf{Q}_{h} \mathbf{u}-\mathbf{u}_{h}\right\|_{1, h} \\
& \lesssim\left\|\mathbf{u}-\mathbf{Q}_{h} \mathbf{u}\right\|_{1, h}+\left|\left\|\mathbf{Q}_{h} \mathbf{u}-\mathbf{u}_{h}\right\|\right| \\
& \lesssim\left\|\mathbf{u}-\mathbf{Q}_{h} \mathbf{u}\right\|_{1, h}+\left|\left\|\mathbf{u}-\mathbf{Q}_{h} \mathbf{u}\right\|\right|+\left|\left\|\mathbf{u}-\mathbf{u}_{h}\right\|\right| .
\end{aligned}
$$

The desired result then follows from the above estimate combined with Lemma 3.4 and Theorem 3.5. 
Theorem 3.7. Suppose that $\Omega$ is a convex bounded polygon or polyhedron. Let $(\boldsymbol{\sigma}, \mathbf{u}) \in$ $\left(H^{s+1}(\Omega)\right)_{d \times d}^{s} \times H^{s+2}(\Omega)^{d}$, where sis a nonnegative integer and $\mathbf{u}$ is the solution of (2.1). Let $\mathbf{u}_{h} \in \mathbf{V}_{h}$ be the solution of (3.7). Then one has

$$
\left\|\mathbf{u}-\mathbf{u}_{h}\right\|_{0} \lesssim h^{\min \{1, k\}+\min \{s+1, k, l+1\}}\|\mathbf{u}\|_{s+2} .
$$

Proof. The proof relies on the usual duality argument. Let $(\widetilde{\boldsymbol{\sigma}}, \widetilde{\mathbf{u}})$ be the solution of the auxiliary problem

$$
\begin{gathered}
\mathcal{A} \widetilde{\boldsymbol{\sigma}}-\boldsymbol{\varepsilon}(\widetilde{\mathbf{u}})=\mathbf{0} \quad \text { in } \Omega, \\
-\nabla \cdot \widetilde{\boldsymbol{\sigma}}=\mathbf{u}-\mathbf{u}_{h} \quad \text { in } \Omega, \\
\widetilde{\mathbf{u}}=\mathbf{0} \quad \text { on } \partial \Omega .
\end{gathered}
$$

Formally, (3.31) is problem (2.1) with $\mathbf{f}$ replaced by $\mathbf{u}-\mathbf{u}_{h}$. Since $\Omega$ is a convex bounded polygon or polyhedron, we have an $H^{2}$-regularity estimate (cf. [28])

$$
\|\tilde{\mathbf{u}}\|_{2} \lesssim\left\|\mathbf{u}-\mathbf{u}_{h}\right\|_{0}
$$

Therefore, applying an elementwise integration by parts to the second equation of (3.31) with a test function $\mathbf{u}-\mathbf{u}_{h}$ and using the definitions of $\mathbf{r}$ (cf. (3.1)) and $a_{h}(\cdot, \cdot)$, the first equation of (3.31), and the technique to derive (2.21), we get

$$
\begin{aligned}
\left\|\mathbf{u}-\mathbf{u}_{h}\right\|_{0}^{2}= & -\sum_{K \in \tau_{h}} \int_{K}\left(\mathbf{u}-\mathbf{u}_{h}\right) \cdot(\nabla \cdot \tilde{\boldsymbol{\sigma}}) d x \\
= & \sum_{K \in \tau_{h}} \int_{K} \tilde{\boldsymbol{\sigma}}: \boldsymbol{\varepsilon}\left(\mathbf{u}-\mathbf{u}_{h}\right) d x-\int_{\varepsilon_{h}} \tilde{\boldsymbol{\sigma}}: \llbracket \mathbf{u}-\mathbf{u}_{h} \rrbracket d s \\
= & a_{h}\left(\widetilde{\mathbf{u}}, \mathbf{u}-\mathbf{u}_{h}\right)-\sum_{K \in \mathcal{\tau}_{h}} \int_{K} \tilde{\boldsymbol{\sigma}}: r\left(\llbracket \mathbf{u}-\mathbf{u}_{h} \rrbracket\right) d x-\int_{\varepsilon_{h}} \tilde{\boldsymbol{\sigma}}: \llbracket \mathbf{u}-\mathbf{u}_{h} \rrbracket d s \\
= & a_{h}\left(\mathbf{u}-\mathbf{u}_{h}, \tilde{\mathbf{u}}\right)-\int_{\varepsilon_{h}}\left\{\tilde{\boldsymbol{\sigma}}-\mathbf{P}_{h} \tilde{\boldsymbol{\sigma}}\right\}: \llbracket \mathbf{u}-\mathbf{u}_{h} \rrbracket d s \\
& -\sum_{K \in \tau_{h}} \int_{K}\left(\tilde{\boldsymbol{\sigma}}-\mathbf{P}_{h} \tilde{\boldsymbol{\sigma}}\right): \mathbf{r}\left(\llbracket \mathbf{u}-\mathbf{u}_{h} \rrbracket\right) d x,
\end{aligned}
$$


and with the identity (3.13), we further have

$$
\begin{aligned}
\left\|\mathbf{u}-\mathbf{u}_{h}\right\|_{0}^{2}= & a_{h}\left(\mathbf{u}-\mathbf{u}_{h}, \tilde{\mathbf{u}}-\mathbf{Q}_{h} \tilde{\mathbf{u}}\right)+a_{h}\left(\mathbf{u}-\mathbf{u}_{h}, \mathbf{Q}_{h} \tilde{\mathbf{u}}\right)-\int_{\varepsilon_{h}}\left\{\tilde{\boldsymbol{\sigma}}-\mathbf{P}_{h} \tilde{\boldsymbol{\sigma}}\right\}: \llbracket \mathbf{u}-\mathbf{u}_{h} \rrbracket d s \\
& -\sum_{K \in \mathcal{T}_{h}} \int_{K}\left(\widetilde{\boldsymbol{\sigma}}-\mathbf{P}_{h} \tilde{\boldsymbol{\sigma}}\right): \mathbf{r}\left(\llbracket \mathbf{u}-\mathbf{u}_{h} \rrbracket\right) d x \\
= & a_{h}\left(\mathbf{u}-\mathbf{u}_{h}, \tilde{\mathbf{u}}-\mathbf{Q}_{h} \tilde{\mathbf{u}}\right)+\int_{\varepsilon_{h}} \llbracket \mathbf{Q}_{h} \tilde{\mathbf{u}}-\tilde{\mathbf{u}} \rrbracket:\left\{\boldsymbol{\sigma}-\mathbf{P}_{h} \boldsymbol{\sigma}\right\} d s \\
& +\sum_{K \in \mathcal{T}_{h}} \int_{K}\left(\boldsymbol{\sigma}-\mathbf{P}_{h} \boldsymbol{\sigma}\right): \mathbf{r}\left(\llbracket \mathbf{Q}_{h} \tilde{\mathbf{u}}-\tilde{\mathbf{u}} \rrbracket\right) d x \\
& -\int_{\varepsilon_{h}}\left\{\tilde{\boldsymbol{\sigma}}-\mathbf{P}_{h} \tilde{\boldsymbol{\sigma}}\right\}: \llbracket \mathbf{u}-\mathbf{u}_{h} \rrbracket d s-\sum_{K \in \tau_{h}} \int_{K}\left(\tilde{\boldsymbol{\sigma}}-\mathbf{P}_{h} \tilde{\boldsymbol{\sigma}}\right): \mathbf{r}\left(\llbracket \mathbf{u}-\mathbf{u}_{h} \rrbracket\right) d x .
\end{aligned}
$$

Therefore, it follows from the boundedness (3.18), the regularity (3.31), and Lemmas 3.1 and 3.4 that

$$
\begin{aligned}
\left\|\mathbf{u}-\mathbf{u}_{h}\right\|_{0}^{2} \lesssim & \left|\left\|\mathbf{u}-\mathbf{u}_{h}\right\|\right|||\left\|\tilde{\mathbf{u}}-\mathbf{Q}_{h} \tilde{\mathbf{u}}\right\||+h|\left\|\mathbf{u}-\mathbf{u}_{h}\right\| \mid\|\tilde{\boldsymbol{\sigma}}\|_{1} \\
& +h^{\min \{s+1, l+1\}}\|\mathbf{u}\|_{s+2}\left(\sum_{e \in \mathcal{E}_{h}} h_{e}^{-1}\left\|\llbracket \mathbf{Q}_{h} \widetilde{\mathbf{u}}-\tilde{\mathbf{u}} \rrbracket\right\|_{0, e}^{2}\right)^{1 / 2} \\
\lesssim & h^{\min \{1, k\}}\left(\left|\left\|\mathbf{u}-\mathbf{u}_{h}\right\|\right|+h^{\min \{s+1, l+1\}}\|\mathbf{u}\|_{s+2}\right)\|\tilde{\mathbf{u}}\|_{2} \\
\lesssim & h^{\min \{1, k\}}\left(\left|\left\|\mathbf{u}-\mathbf{u}_{h}\right\|\right|+h^{\min \{s+1, l+1\}}\|\mathbf{u}\|_{s+2}\right)\left\|\mathbf{u}-\mathbf{u}_{h}\right\|_{0}
\end{aligned}
$$

This, along with Theorem 3.5, immediately leads to

$$
\left\|\mathbf{u}-\mathbf{u}_{h}\right\|_{0} \lesssim h^{\min \{1, k\}}\left(\left|\left\|\mathbf{u}-\mathbf{u}_{h}\right\|\right|+h^{\min \{s+1, l+1\}}\|\mathbf{u}\|_{s+2}\right) \lesssim h^{\min \{1, k\}+\min \{s+1, k, l+1\}}\|\mathbf{u}\|_{s+2} .
$$

We would like to end this section with some results on the optimality of our estimates derived. If we choose $S_{1}(K)$ and $S_{2}(K)$ to be $P_{l}(K)$ and $P_{k}(K)$, respectively, it is easy to check that the first condition of (2.5) implies $k-1 \leq l$. Therefore, we can obtain the following result from the previous theorems directly.

Corollary 3.8. Let $(\boldsymbol{\sigma}, \mathbf{u}) \in\left(H^{s+1}(\Omega)\right)_{d \times d}^{s} \times H^{s+2}(\Omega)^{d}$, where $s$ is a nonnegative integer and $\mathbf{u}$ is the solution of (2.1). Let $\mathbf{u}_{h} \in \mathbf{V}_{h}$ be the solution of (3.7) with $S_{1}(K)=P_{l}(K)$ and $S_{2}(K)=P_{k}(K)$ for all $K \in \tau_{h}$. Then one has the following optimal error estimates:

$$
\begin{aligned}
\left\|\left|\mathbf{u}-\mathbf{u}_{h}\right|\right\| & \lesssim h^{\min \{s+1, k\}}\|\mathbf{u}\|_{s+2}, \\
\left\|\mathbf{u}-\mathbf{u}_{h}\right\|_{1, h} & \lesssim h^{\min \{s+1, k\}}\|\mathbf{u}\|_{s+2} .
\end{aligned}
$$




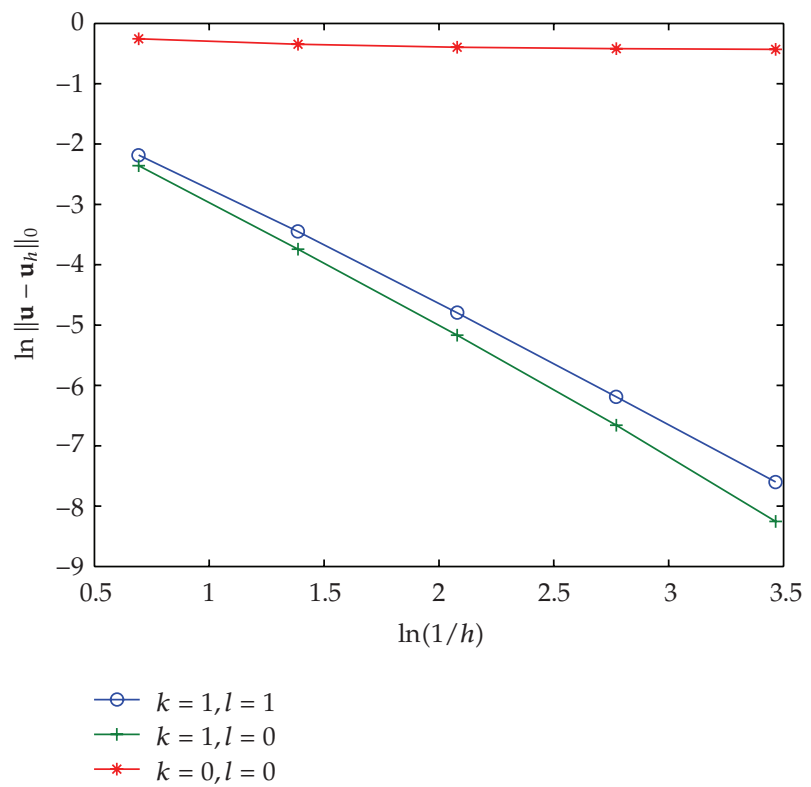

Figure 1: $\left\|\mathbf{u}-\mathbf{u}_{h}\right\|_{0}$ in the $\ln -\ln$ scale for several choices of $k$ and $l$.

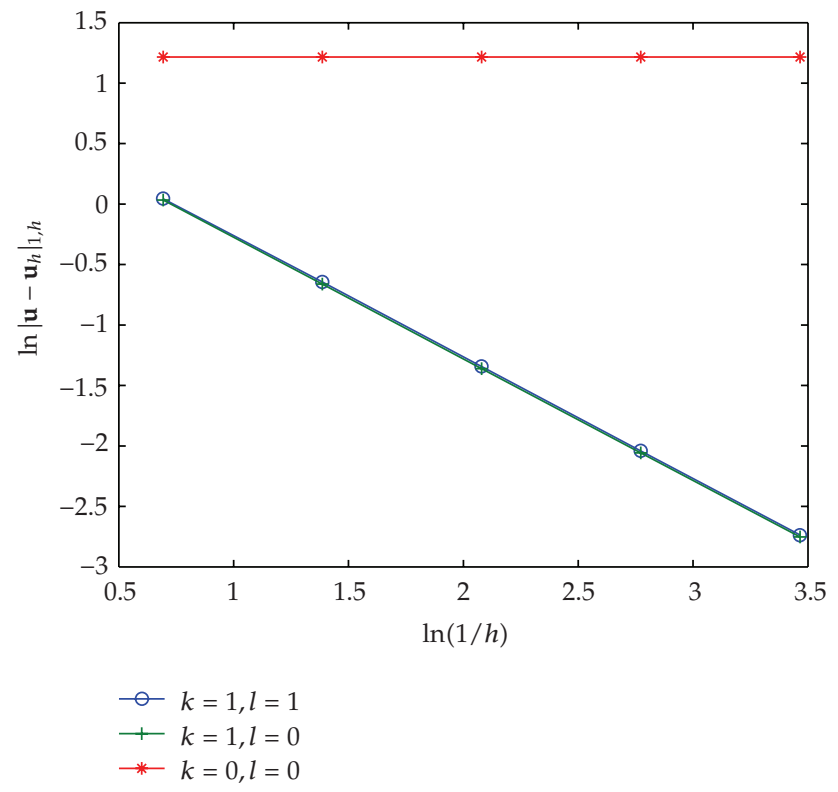

Figure 2: $\left|\mathbf{u}-\mathbf{u}_{h}\right|_{1, h}$ in the $\ln -\ln$ scale for several choices of $k$ and $l$.

In addition, if $\Omega$ is a convex bounded polygon or polyhedron,

$$
\left\|\mathbf{u}-\mathbf{u}_{h}\right\|_{0} \lesssim h^{\min \{1, k\}+\min \{s+1, k\}}\|\mathbf{u}\|_{s+2} .
$$




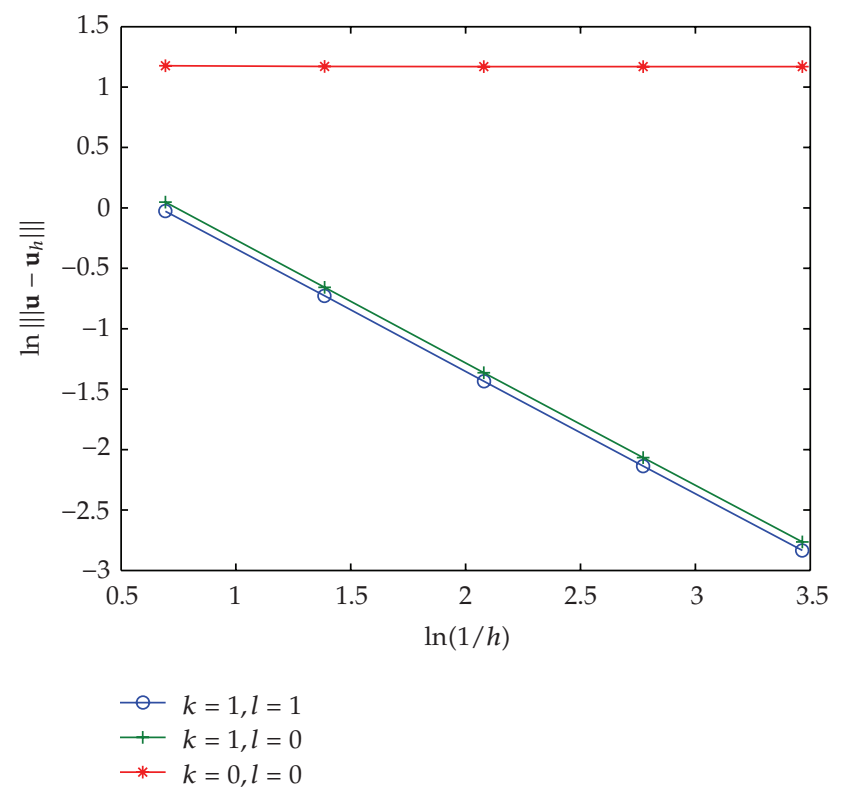

Figure 3: $\left\|\mathbf{u}-\mathbf{u}_{h}\right\| \mid$ in the $\ln -\ln$ scale for several choices of $k$ and $l$.

\section{Numerical Results}

In this section, we report a numerical example to illustrate the theoretical results. Let $\Omega=$ $(-1,1) \times(-1,1), \lambda=0.3, \mu=0.35$, and

$$
\begin{aligned}
\mathbf{f}\left(x_{1}, x_{2}\right)= & 2 \mu\left(3-x_{1}^{2}-2 x_{2}^{2}-2 x_{1} x_{2}, 3-2 x_{1}^{2}-x_{2}^{2}-2 x_{1} x_{2}\right)^{t} \\
& +\lambda\left(2-2 x_{2}^{2}-4 x_{1} x_{2}, 2-2 x_{1}^{2}-4 x_{1} x_{2}\right)^{t} .
\end{aligned}
$$

It is not difficult to check that the exact solution of (2.1) is

$$
\mathbf{u}\left(x_{1}, x_{2}\right)=\left(\left(1-x_{1}^{2}\right)\left(1-x_{2}^{2}\right),\left(1-x_{1}^{2}\right)\left(1-x_{2}^{2}\right)\right)^{t} .
$$

We use a quasiuniform triangulation $\tau_{h}$ over $\Omega$. For any $K \in \tau_{h}$, we take $S_{1}(K)=P_{l}(K)$ and $\mathcal{S}_{2}(K)=P_{k}(K)$ where $k, l \geq 0$. On each edge $e, \eta_{e}$ is set to be 1 . The numerical results of the LDG method for a few choices of $k$ and $l$ are shown in Table 1 and Figures 1, 2, and 3. We observe that, when $k=1$, the numerical convergence rates of $\left\|\mathbf{u}-\mathbf{u}_{h}\right\|_{0},\left|\mathbf{u}-\mathbf{u}_{h}\right|_{1, h}$, and $\left|\left\|\mathbf{u}-\mathbf{u}_{h}\right\|\right|$ are $O\left(h^{2}\right), O(h)$, and $O(h)$, respectively. However, there is no convergence when $k=0$. These phenomena agree with theoretical results in Theorems 3.5-3.7. When $k=1$, accuracies of the numerical results are nearly the same as those for $l=0$ and $l=1$. It goes without saying that it is more convenient to simulate for $l=0$ than for $l=1$. 
Table 1: Numerical errors of LDG method for several choices of $k$ and $l$. The theoretical convergence rates of $\left\|\mathbf{u}-\mathbf{u}_{h}\right\|_{0},\left|\mathbf{u}-\mathbf{u}_{h}\right|_{1, h}$, and $\|\left|\mathbf{u}-\mathbf{u}_{h}\right|||$ are $O\left(h^{k+\min \{1, k\}}\right), O\left(h^{k}\right)$, and $O\left(h^{k}\right)$, respectively.

\begin{tabular}{|c|c|c|c|c|c|c|}
\hline$k, l$ & $h$ & $2^{-1}$ & $2^{-2}$ & $2^{-3}$ & $2^{-4}$ & $2^{-5}$ \\
\hline \multirow[t]{3}{*}{$k=1, l=1$} & $\left\|\mathbf{u}-\mathbf{u}_{h}\right\|_{0}$ & 0.11271 & 0.03173 & 0.00827 & 0.00205 & 0.00050 \\
\hline & $\left|\mathbf{u}-\mathbf{u}_{h}\right|_{1, h}$ & 1.04389 & 0.52475 & 0.26098 & 0.12990 & 0.06477 \\
\hline & $\left\|\mathbf{u}-\mathbf{u}_{h}\right\| \mid$ & 0.97179 & 0.48304 & 0.23833 & 0.11806 & 0.05872 \\
\hline \multirow[t]{3}{*}{$k=1, l=0$} & $\left\|\mathbf{u}-\mathbf{u}_{h}\right\|_{0}$ & 0.09448 & 0.02371 & 0.00570 & 0.00128 & 0.00026 \\
\hline & $\left|\mathbf{u}-\mathbf{u}_{h}\right|_{1, h}$ & 1.03341 & 0.51585 & 0.25644 & 0.12773 & 0.06373 \\
\hline & $\left|\left\|\mathbf{u}-\mathbf{u}_{h}\right\|\right|$ & 1.04905 & 0.51847 & 0.25572 & 0.12673 & 0.06305 \\
\hline \multirow[t]{3}{*}{$k=0, l=0$} & $\left\|\mathbf{u}-\mathbf{u}_{h}\right\|_{0}$ & 0.77564 & 0.70692 & 0.67276 & 0.65756 & 0.65067 \\
\hline & $\left|\mathbf{u}-\mathbf{u}_{h}\right|_{1, h}$ & 3.37310 & 3.37310 & 3.37310 & 3.37310 & 3.37310 \\
\hline & $\left|\left\|\mathbf{u}-\mathbf{u}_{h}\right\|\right|$ & 3.24274 & 3.22244 & 3.21906 & 3.21822 & 3.21790 \\
\hline
\end{tabular}

\section{Acknowledgments}

The authors thank the referee for the valuable comments and suggestions, which greatly improved the presentation of the manuscript. The research of the second author is supported partially by the NNSFC (10371076) and E-Institutes of Shanghai Municipal Education Commission (E03004).

\section{References}

[1] B. Cockburn, G. E. Karniadakis, and C.-W. Shu, Eds., Discontinuous Galerkin Methods. Theory, Computation and Applications, vol. 11 of Lecture Notes in Computational Science and Engineering, Springer, Berlin, Germany, 2000.

[2] F. Bassi and S. Rebay, "A high-order accurate discontinuous finite element method for the numerical solution of the compressible Navier-Stokes equations," Journal of Computational Physics, vol. 131, no. 2, pp. 267-279, 1997.

[3] B. Cockburn and C.-W. Shu, "The local discontinuous Galerkin method for time-dependent convection-diffusion systems," SIAM Journal on Numerical Analysis, vol. 35, no. 6, pp. 2440-2463, 1998.

[4] P. Castillo, B. Cockburn, I. Perugia, and D. Schötzau, "An a priori error analysis of the local discontinuous Galerkin method for elliptic problems," SIAM Journal on Numerical Analysis, vol. 38, no. 5, pp. 1676-1706, 2000.

[5] D. N. Arnold, F. Brezzi, B. Cockburn, and L. D. Marini, "Unified analysis of discontinuous Galerkin methods for elliptic problems," SIAM Journal on Numerical Analysis, vol. 39, no. 5, pp. 1749-1779, $2001 / 02$.

[6] F. Brezzi, B. Cockburn, L. D. Marini, and E. Süli, “Stabilization mechanisms in discontinuous Galerkin finite element methods," Computer Methods in Applied Mechanics and Engineering, vol. 195, no. 25-28, pp. 3293-3310, 2006.

[7] B. Cockburn, "Discontinuous Galerkin methods," Zeitschrift für Angewandte Mathematik und Mechanik, vol. 83, no. 11, pp. 731-754, 2003.

[8] D. N. Arnold and R. Winther, "Mixed finite elements for elasticity," Numerische Mathematik, vol. 92, no. 3, pp. 401-419, 2002.

[9] S. Adams and B. Cockburn, "A mixed finite element method for elasticity in three dimensions," Journal of Scientific Computing, vol. 25, no. 3, pp. 515-521, 2005.

[10] B. M. Fraeijs de Veubeke, "Displacement and equilibrium models the finite element method," in Stress Analysis, O. C. Zienkiewicz and G. S. Holister, Eds., pp. 145-197, Wiley, New York, NY, USA, 1965.

[11] V. B. Watwood Jr. and B. J. Hartz, "An equilibrium stress field model for finite element solution of two-dimensional elastostatic problems," International Journal of Solids and Structures, vol. 4, pp. 857$873,1968$.

[12] C. Johnson and B. Mercier, "Some equilibrium finite element methods for two-dimensional elasticity problems," Numerische Mathematik, vol. 30, no. 1, pp. 103-116, 1978. 
[13] D. N. Arnold, J. Douglas Jr., and C. P. Gupta, "A family of higher order mixed finite element methods for plane elasticity," Numerische Mathematik, vol. 45, no. 1, pp. 1-22, 1984.

[14] D. N. Arnold, F. Brezzi, and J. Douglas, Jr., "PEERS: a new mixed finite element for plane elasticity," Japan Journal of Applied Mathematics, vol. 1, no. 2, pp. 347-367, 1984.

[15] D. N. Arnold, R. S. Falk, and R. Winther, "Mixed finite element methods for linear elasticity with weakly imposed symmetry," Mathematics of Computation, vol. 76, no. 260, pp. 1699-1723, 2007.

[16] W. Qiu and L. Demkowicz, "Mixed $h b$-finite element method for linear elasticity with weakly imposed symmetry," Computer Methods in Applied Mechanics and Engineering, vol. 198, no. 47-48, pp. 3682-3701, 2009.

[17] A. Lew, P. Neff, D. Sulsky, and M. Ortiz, “Optimal BV estimates for a discontinuous Galerkin method for linear elasticity," Applied Mathematics Research eXpress, vol. 2004, no. 3, pp. 73-106, 2004.

[18] P. Hansbo and M. G. Larson, "Discontinuous Galerkin methods for incompressible and nearly incompressible elasticity by Nitsche's method," Computer Methods in Applied Mechanics and Engineering, vol. 191, no. 17-18, pp. 1895-1908, 2002.

[19] P. Hansbo and M. G. Larson, "Discontinuous Galerkin and the Crouzeix-Raviart element: application to elasticity," M2AN. Mathematical Modelling and Numerical Analysis, vol. 37, no. 1, pp. 63-72, 2003.

[20] Z. Cai and X. Ye, "A mixed nonconforming finite element for linear elasticity," Numerical Methods for Partial Differential Equations, vol. 21, no. 6, pp. 1043-1051, 2005.

[21] R. Bustinza, "A note on the local discontinuous Galerkin method for linear problems in elasticity," Scientia Series A, vol. 13, pp. 72-83, 2006.

[22] K. Feng and Z. Shi, Mathematical Theory of Elastic Structures, Springer, Berlin, Germany, 1995.

[23] S. C. Brenner and L. R. Scott, The Mathematical Theory of Finite Element Methods, vol. 15 of Texts in Applied Mathematics, Springer, New York, NY, USA, 3rd edition, 2008.

[24] P. G. Ciarlet, The Finite Element Method for Elliptic Problems, vol. 4 of Studies in Mathematics and Its Applications, North-Holland, Amsterdam, The Netherlands, 1978.

[25] G. Fichera, "Existence theorems in elasticity," in Handbuch der Physik, Band VI, pp. 347-389, Springer, Heidelberg, Germany, 1972.

[26] S. C. Brenner, "Korn's inequalities for piecewise $H^{1}$ vector fields," Mathematics of Computation, vol. 73, no. 247, pp. 1067-1087, 2004.

[27] F. Brezzi and M. Fortin, Mixed and Hybrid Finite Element Methods, vol. 15 of Springer Series in Computational Mathematics, Springer, New York, NY, USA, 1991.

[28] P. Grisvard, Singularities in Boundary Value Problems, vol. 22 of Recherches en Mathématiques Appliquées, Masson, Paris, France; Springer, Berlin, Germany, 1992. 


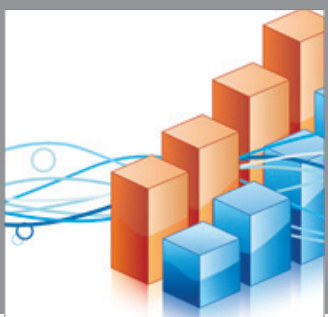

Advances in

Operations Research

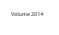

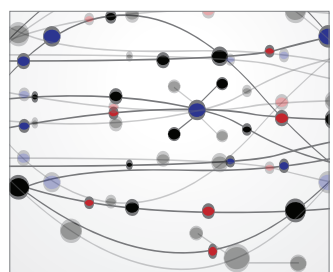

\section{The Scientific} World Journal
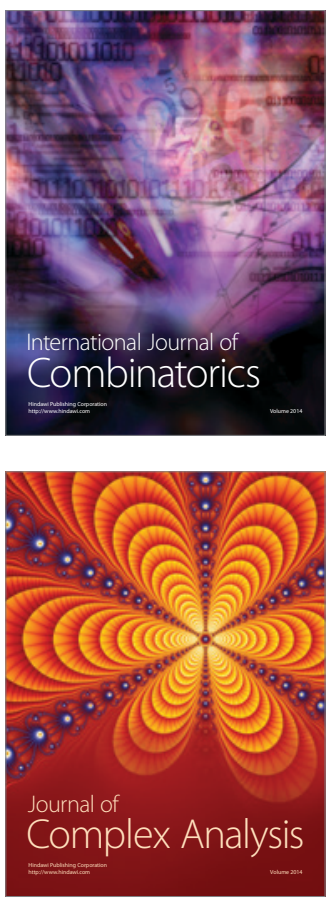

International Journal of

Mathematics and

Mathematical

Sciences
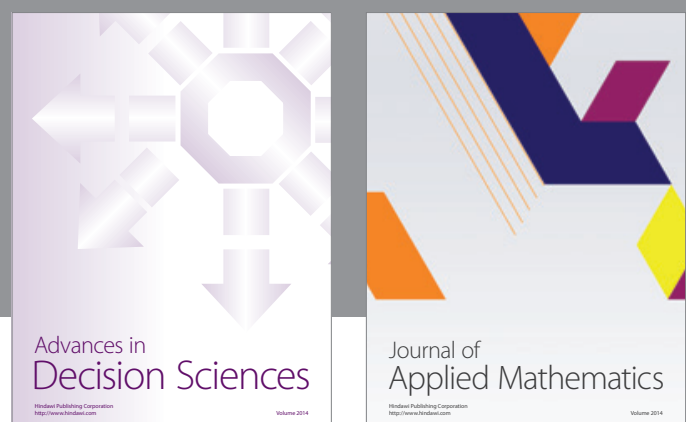

Journal of

Applied Mathematics
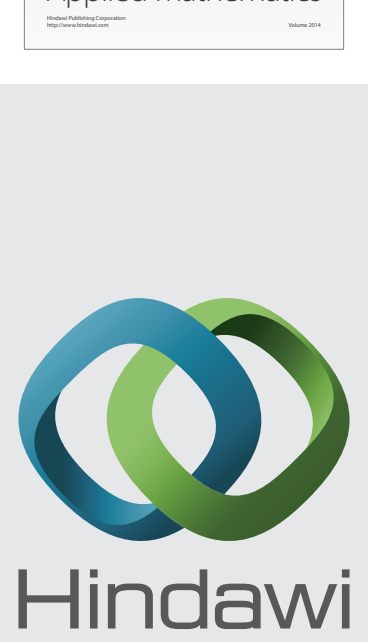

Submit your manuscripts at http://www.hindawi.com
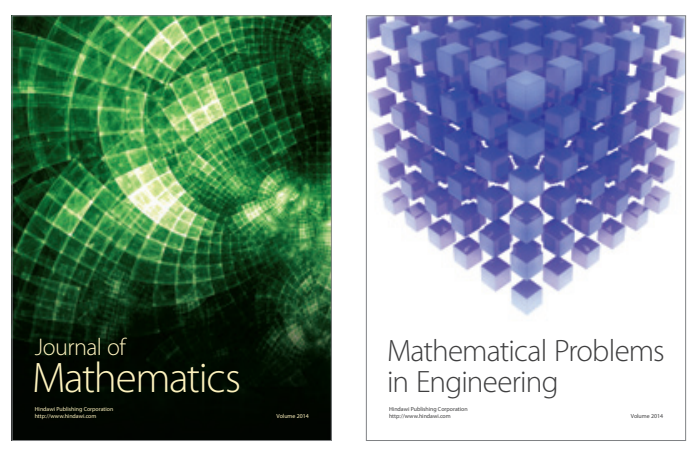

Mathematical Problems in Engineering
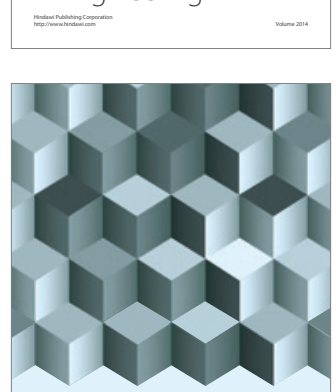

Journal of

Function Spaces
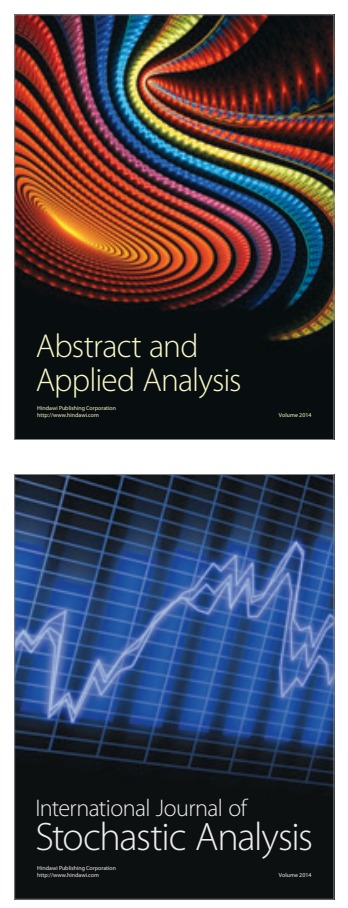

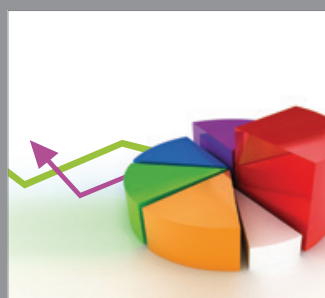

ournal of

Probability and Statistics

Promensencen
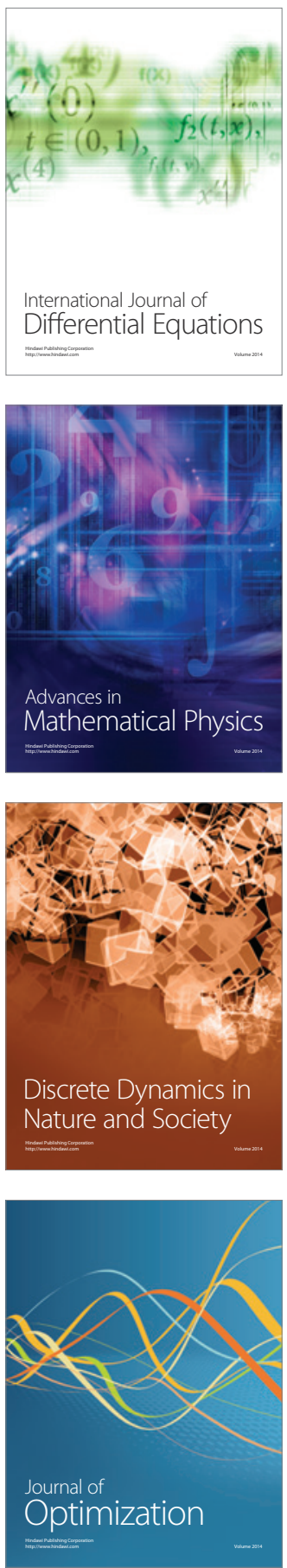